\title{
Estratégias autorregulatórias para o controle do desempenho em estudantes universitários: mudanças após processo reflexivo
}

\section{Self-regulating strategies for performance control in university students: changes after reflexive process}

\author{
Célia Artemisa Gomes Rodrigues Miranda, Amélia Rodrigues Nonticuri, Lourdes Maria Bragagnolo Frison \\ Universidade Federal de Pelotas
}

\begin{abstract}
Resumo
Este artigo objetiva apresentar as estratégias autorregulatórias que os estudantes universitários sinalizaram que efetivamente utilizam (Real) para o controle do desempenho, comparando com aquelas que consideram mais relevantes para melhorar sua aprendizagem (Ideal). Além disso, pontuamos as percepções em relação à escolha destas estratégias. Participaram 19 estudantes brasileiros, que marcaram no Questionário de Estratégias de Autorregulação do Controle do Desempenho - (QEACD Real-Ideal), a estratégia da Gestão da atenção (79\%). As mudanças mais expressivas se deram nas estratégias das dimensões Pedir ajuda e Gestão emocional/da ansiedade, que após processo reflexivo gerado pela pesquisa, decidiram adotar, conforme relatos da entrevista final.

Palavras chave: controle do desempenho, autorregulação, universitários.
\end{abstract}

\begin{abstract}
This article aims to present the self-regulatory strategies that university students have signalled that effectively use (Real) for performance control, comparing them with those they consider most relevant to improve their learning (Ideal). In addition, we punctuate the perceptions regarding the choice of these strategies. Nineteen Brazilian students marked in the Strategy Management Self-Regulation Questionnaire (QEACD Real-Ideal), the strategy of the Management of attention (79\%). The most expressive changes were at the dimensions Ask for help and Emotional / anxiety management, which after the reflexive process generated by the research, decided to adopt, as reported in the final interview.

Keywords: performance control, self-regulation, university students.
\end{abstract}

\section{Introdução}

Os problemas que os estudantes enfrentam ao entrarem no ensino universitário, advém, muitas vezes, de não saberem mobilizar as estratégias adequadas para a sua aprendizagem. $\mathrm{O}$ contraste de uma aprendizagem mais paternalista que se verifica antes da entrada na universidade, monitorada essencialmente pelos adultos sobre o quê, quando ou como estudar, acaba por subsumir a aprendizagem autônoma do estudante, uma vez que eles precisam ter consciência e controle sobre seu próprio aprender. Essa autonomia desejada, pouco foi exigida ou acompanhada, estimulada no percurso da escola básica, o que dificulta o desenvolvimento autorregulatório dos próprios estudantes para gerirem sua aprendizagem. Desse modo, os problemas que os estudantes enfrentam ao entrarem no ensino universitário, decorre também da falta de conhecimento de determinadas estratégias autorregulatórias, ou do uso limitado e inadequado que fazem delas.

A autorregulação da aprendizagem, longe de ser uma habilidade mental, como a inteligência, é antes de tudo um processo autodirigido em que os alunos transformam as habilidades mentais em competências acadêmicas (Zimmerman, 1998; 2000). Desse modo, por meio da Autorregulação da aprendizagem os estudantes desenvolvem autonomia para expressar as competências com as quais possam gerenciar e monitorar conscientemente a sua cognição, motivação e comportamento com o intuito de alcançar os objetivos propostos para a sua aprendizagem (Veiga Simão; Frison, 2013; Zimmerman, 1986; 2000). Esse processo multidimensional, envolve variáveis pessoais, como os aspectos cognitivos, motivacionais, comportamentais e contextuais (Zimmerman, 1986, 1989) que guiam os estudantes a fazerem escolhas estratégicas para organizar, planejar, controlar e avaliar seu processo de aprendizagem (Veiga Simão, 2004, Serafim; Boruchovich, 2010). A autorregulação da aprendizagem requer do estudante o seu envolvimento nas três fases cíclicas, as quais se relacionam entre si: antecipação, execução ou controle volitivo e autorreflexão (Figura 1).

A fase de Antecipação envolve o planejamento e a preparação dos esforços para aprender. Os estudantes devem levar em conta as influências, crenças motivacionais, metas e estratégias envolvidos numa determinada atividade. As estratégias criadas pelos alunos devem considerar o planejamento e ajuste segundo suas condições pessoais, comportamentais e ambientais. A fase de Execução ou controle volitivo, refere-se aos esforços e estratégias utilizadas durante a aprendizagem, com a intenção de facilitar a concentração, controle e monitoramento da volição para alcançar os objetivos estabelecidos na fase de 
Antecipação. Deve ser planejada na Fase de Antecipação considerando a capacidade do aluno de autocontrolar e de se auto-observar durante o processo de aprendizagem.

A fase de autorreflexão, abrange os esforços empreendidos após a aprendizagem e influenciam as reações dos estudantes frente aos resultados alcançados. Ocorre por meio do autojulgamento e da autorreação. Vale referir que cada uma das fases influencia as fases precedentes e os passos seguintes para alcançar a aprendizagem, completando deste modo o ciclo autorregulatório (ZIMMERMAN, 1998, 2000, 2013).

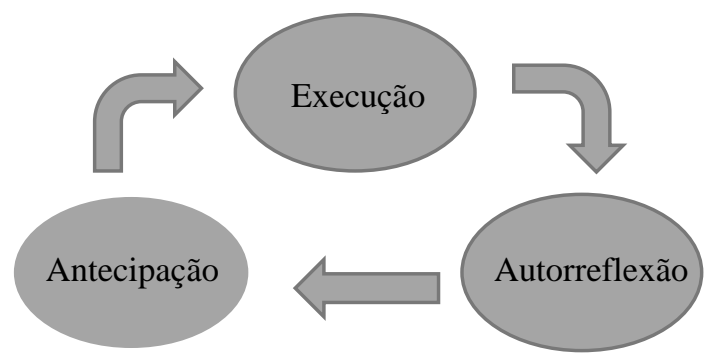

Figura 1: Fases cíclicas da aprendizagem acadêmica (Zimmerman, 1986; 2013).

A partir da compreensão das fases da autorregulação, podemos dizer que o aluno que autorregula sua aprendizagem é aquele que potencializa seu esforço durante o estudo e consegue mobilizar adequadamente estratégias autorregulatórias para a sua aprendizagem de forma cíclica. As estratégias de aprendizagem, diferente das técnicas de estudo, podem ser definidas como um conjunto de atividades mentais que o indivíduo mobiliza de modo consciente e intencional para facilitar a aquisição do conhecimento (Veiga Simão, 2013). Saber como, quando e por que utilizar essas estratégias, tem sido apontada por muitos pesquisadores como essenciais para a potencialização da aprendizagem e um bom desempenho acadêmico (Boruchovich, 1999; 2007; Buzuneck, 2004, Pozo, 2008; Rosa; Deps, 2015; Serafim; Boruchovich, 2010; Veiga Simão, 2013; Zimmerman, 2013). Portanto, durante a realização de uma atividade, a escolha das estratégias deve levar em conta o tipo de objetivo que se pretende atingir.

As estratégias de controle, ou estratégias volitivas, requerem do estudante um esforço continuo para manterem concentrados na atividade, evitando estímulos distratores que possam atrapalhar os objetivos da aprendizagem (Carta, 2012; Corno, 2001; Deps; Veiga Simão; Lopes Da Silva, 2011; Rosa; Deps, 2015).

Diversas investigações têm sido feitas no sentido de elucidar as estratégias autorregulatórias mais utilizadas pelos estudantes em seus estudos (Medeiros, 2014; Perrassinato, Boruchovich; Oliveira, 2012; Miranda, Nonticuri, Louzada, Frison, 2016; Veiga Simão, Frison, Nonticuri, 2015; Zimmerman; Martinez-Pons, 1986) ressaltando a importância de conhecer as estratégias utilizadas pelos estudantes para melhor planejar intervenções que os auxiliem a aprimorar essas estratégias.

Zimmerman e Martinez-Pons (1986), ao avaliar os processos da autorregulação da aprendizagem de uma grande amostra de estudantes, identificam 14 estratégias essenciais: 1- autoavaliação, 2- organização e transformação da informação, 3- estabelecimento de metas e planejamento, 4- procura da informações, 5manter registro e monitoramento, 6- estrutura do ambiente, 7- autoconsequências, 8- ensaio e memorização, 9 a 11- procura por ajuda social (professores, adultos, pares), 12 a 14 - revisar anotações (anotações, livros textos e reler teste) e 15- outras e, constataram que o uso destas estratégias está altamente correlacionado a um bom desempenho escolar.

Assim, este artigo objetiva apresentar as estratégias autorregulatórias que os estudantes universitários realmente utilizam para controlar o seu desempenho durante a realização de uma atividade de estudo, contrapondo com as estratégias que consideram mais relevantes para alcançar melhorares resultados em sua aprendizagem, além das percepções em relação às estratégias escolhidas para aferir se houveram mudanças.

\section{Metodologia}

Este trabalho integra o projeto de investigação "Dos significados à autorregulação: perspectivas de estudantes com trajetórias acadêmicas de insucesso" realizado pela Universidade Federal de Pelotas (Brasil) em parceria com a Universidade de Lisboa (Portugal). Neste artigo apresentamos um recorte dos resultados alcançados no Brasil, que contou com a participação de 19 estudantes universitários, amparados pelo Programa de Assistência Estudantil da universidade (bolsistas da universidade), que apresentavam desempenho acadêmico inferior a $70 \%$ de aprovação nas disciplinas matriculadas. A coleta dos dados contou com uma metodologia diversificada, fazendo uso de entrevista inicial e final, aplicação de questionários e escritas de uma narrativa autobiográfica. No primeiro encontro foi realizada uma entrevista semiestruturada que buscou caracterizar e conhecer os estudantes. Também foi solicitado que escrevessem uma narrativa autobiográfica. No segundo encontro, para identificar as estratégias que estes estudantes utilizavam no seu processo de aprendizagem, foi solicitado que respondessem a Escala de Autorregulação da Motivação para a Aprendizagem - EAMA (Paulino; Lopes Da Silva; Sá, 2015) e ao Questionário de Estratégias de Autorregulação do Controle do Desempenho - QEACD (Veiga Simão; Lopes da Silva, 2012), marcando na escala Likert de 5 itens $^{1}$, a frequência com que executavam as estratégias mencionadas, conforme a sua realidade. A esta primeira marcação denominamos EAMA Real e QEACD Real. Logo após, os estudantes sinalizaram dentre as estratégias que constavam nos instrumentos de pesquisa EAMA e QEACD, as que consideravam mais importantes para melhorarem seu desempenho acadêmico, priorizando dez estratégias em cada instrumento. Desta vez sem a escala Likert, considerando

\footnotetext{
${ }^{1}$ Escala de 5 pontos, em que $1=$ nunca, 2 = poucas vezes, $3=$ algumas vezes, $4=$ muitas vezes e $5=$ sempre.
} 
apenas a estratégia em si e não a frequência com que utilizavam. Esta segunda marcação foi denominada de EAMA Ideal e QEACD Ideal. O foco principal deste artigo é pontuar os resultados obtidos em relação ao QAECD Real e Ideal. Este instrumento contém 21 afirmações relativas a autorrelatos de estratégias autorregulatórias para o controle do desempenho, apresentadas na tabela abaixo.

Tabla 1.

Dimensões das estratégias do QEACD.

\begin{tabular}{lc}
\hline Dimensões do QEACD & Estratégias \\
\hline 1- Testar & $1,14,18$ \\
2- Pedir ajuda & $2,12,16$ \\
3- Rever o que foi feito & $3,10,15$ \\
4- Gestão do tempo & $4,8,20$ \\
5- Gestão da atenção & $5,11,19$ \\
6- Gestão emocional da ansiedade & $7,17,21$ \\
7- Avaliação do processo e monitorização & $6,9,13$ \\
do estudo &
\end{tabular}

Fonte: Frison, Veiga Simão, Nonticuri (2015).

Retomando o que já haviam feito, na entrevista final, os estudantes apresentaram suas percepções sobre as estratégias consideradas mais relevantes para o aprendizado, justificando a escolha.

A partir das repostadas dos estudantes, foi realizada uma análise comparativa entre as estratégias que eles consideravam ideais e aquelas que realmente utilizavam, bem como as que não foram citadas em nenhum momento, mas percebidas como imprescindíveis para potencializar o seu processo de aprendizagem.

\section{Resultados e discussão}

A análise foi efetuada, observando quais as estratégias mais assinaladas no contexto Real e Ideal dos estudantes e que mudanças indicaram a partir da marcação das estratégias após refletirem sobre cada uma delas. Estas mudanças podem ser observadas na reflexão dos alunos durante a entrevista realizada após escolha de cada estratégia. Para caracterizar os diferentes depoimentos dos estudantes que fizeram parte da pesquisa, utilizamos a letra $\mathrm{E}$ (estudante), seguida de números de 1 a 19.

Dos resultados obtidos a partir do QEACD (Tabela 2), as estratégias mais sinalizadas e, que fazem muitas vezes e sempre (opção 4 e 5 da escala Likert), referem-se à Gestão da atenção, com $78.95 \%$ das marcações, seguida da gestão emocional/ da ansiedade com 56.14\%. A dimensão menos sinalizada, refere à aquela que apresentam as estratégias da gestão do tempo, o que evidencia a dificuldade dos alunos em gerir o tempo durante o estudo.

Com relação às estratégias que compõem cada uma das dimensões do QEACD - Real, os estudantes apontaram a estratégia 11 (Quando inicio o estudo, penso que tenho de estar concentrado (a) no que estou fazendo), que obteve uma marcação de mais de $84,2 \%$ dos alunos e a estratégia 19 (Enquanto estou estudando tento ignorar o que pode me distrair), com $78,95 \%$ de indicações. As duas estratégias pertencem à dimensão Gestão da atenção.

A Gestão da atenção se destacou como uma dimensão muito presente no cotidiano da vida acadêmica dos estudantes, preocupados em eliminar os distratores e manter o foco nos estudos. Como a literatura aponta, os estudantes tendem a alcançar melhores resultados na aprendizagem, se empenham na realização das tarefas e persistem a possíveis distrações que possam ocorrer (Carta, 2012; Zimmerman, 2000). A partir da análise dos dados do QEACD - Real, observamos que as estratégias que os estudantes mais utilizam revelam o cuidado com a gestão dos distratores, quando buscam eliminá-los ou reduzi-los para manter o foco. Relataram que procuram manter a atenção também no momento das avaliações, buscando revisar as respostas antes de entregar a prova.

Estes resultados reforçam o que encontramos na literatura (Boruchovich, 1999; 2007; Buzuneck, 2004; Rosa; Deps, 2015; Veiga Simão, 2013; Zimmerman, 2013) sobre a importância de estimular o uso de estratégias, com as quais os estudantes podem melhorar a concentração e a gestão de múltiplos distratores. Conforme relatou o estudante E6: Concentração é fundamental para realizar qualquer tarefa, se não estamos concentrados não conseguimos atingir o objetivo. Os estudantes reforçaram que apenas realizar uma tarefa como ler ou escrever sem realmente estarem concentrados no conteúdo da atividade, pouco valerá para seu aprendizado. De acordo com Zimmerman (2000) uma das características do estudante autorregulado é a sua capacidade de manter a atenção e o esforço na realização das tarefas, mesmo quando surgem novos distratores.

Após o momento reflexivo sobre as dimensões implícitas no questionário - QEACD, os estudantes destacaram como as mais importantes, um conjunto de estratégias que comparadas com as que realmente utilizam, não se diferencia muito do que pontuam como ideais. Podemos observar que as marcações se mantiveram constantes, com poucas alterações. Ressaltamos, porém, as dimensões da Gestão emocional da Ansiedade e do Pedir Ajuda, em que ambas sofreram uma variação positiva de $14 \%$ da situação real para o ideal. Assim, podemos pontuar que as principais mudanças nas percepções dos estudantes em relação às estratégias mais importantes para o seu processo de aprendizagem situam-se nessas duas dimensões. Essa realidade denota o anseio dos estudantes em se munirem de estratégias volitivas para alcançar os seus objetivos. 
Tabela 2:

Frequência (freq.) das marcações de cada dimensão

\begin{tabular}{ccccccc}
\hline & \multicolumn{2}{c}{ QEACD } & \multicolumn{2}{c}{ QEACD } & Ordem & Variação \\
\hline Dimensão & Freq & $\%$ & Freq & $\%$ & Ideal & $\begin{array}{c}\text { Ideal - } \\
\text { real }\end{array}$ \\
5 & 45 & 79.0 & 41 & 71.9 & $1^{\circ}$ & -7.1 \\
6 & 32 & 56.1 & 40 & 70.1 & $2^{\circ}$ & 14 \\
2 & 28 & 49.1 & 36 & 63.2 & $3^{\circ}$ & 14,1 \\
3 & 27 & 47.4 & 23 & 40.4 & $4^{\circ}$ & -7 \\
1 & 24 & 42.1 & 19 & 33.4 & $5^{\circ}$ & -8.7 \\
4 & 19 & 33.3 & 16 & 28.1 & $6^{\circ}$ & -5.2 \\
7 & 26 & 45.6 & 15 & 26.3 & $7^{\circ}$ & -19.3 \\
\hline
\end{tabular}

As estratégias relacionadas às dimensões Avaliação do processo e Avaliação da monitorização do estudo, embora identificadas pelos estudantes como aquelas que realizam sistematicamente, não foram tão marcadas quando pensadas numa situação ideal para um aluno autorregulado. A variação foi de $19.3 \%$ negativos em relação ao Real. Entendem que é mais importante as estratégias relacionadas às dimensões Gestão emocional da ansiedade, Pedir ajuda e Testar. Demostraram ter maior ênfase na estratégia 2 (Quando tenho dúvidas durante meu estudo, peço ajuda aos meus colegas), o que reforça a participação de seus colegas no processo de aprendizagem. Esta estratégia é apontada por autores como tendo um papel relevante na aprendizagem autorregulada, pois para pedir ajuda, o estudante deve estar consciente dos seus desafios e dificuldades, mobilizando recursos internos para determinar quando, como e para quem pedir ajuda (Serafim; Boruchovich, 2010). Durante o processo reflexivo proporcionado pela pesquisa, esta estratégia foi repensada pelos estudantes e passou a ser valorizada como uma ação importante para a melhoria de seu desempenho. Conforme alguns depoimentos: Com o professor a gente não entende com os colegas às vezes é mais fácil, com uma linguagem mais fácil (E18); os colegas podem ter perguntas parecidas que eles conseguem explicar muito melhor (E12), percebemos que muitos já realizavam essa estratégia e pontuaram algumas vantagens, como uma maior facilidade para o entendimento dos conteúdos, quando explicados ou problematizados por outros colegas

\section{Conclusões}

Este estudo apresenta limitações devido ao baixo número de participantes, o que impede ou não nos permite fazer inferências estatísticas com a finalidade de fazer generalizações sobre os dados encontrados, entretanto, permite sinalizar estratégias importantes que os alunos deixam de lado, que poderiam auxiliar na melhoria da aprendizagem, como o pedir ajuda quando necessário. Também, é preciso estar atento à percepção que os estudantes têm de que o sucesso do estudo está relacionado apenas ao esforço e desempenho deles durante a aprendizagem, centrando seus esforços apenas em realizar todas as tarefas propostas pelos professores. Estes dados podem ser considerados em estudos futuros que possam vir a investir no mesmo gênero de pesquisa. Além do mais, os resultados encontrados no Brasil se aproximam dos revelados pelos estudantes de Portugal, os quais tiveram o mesmo procedimento de recolha e tratamento de dados.

\section{Referencias}

Buzuneck, J. A. (2004). Aprendizagem por processamento da informação: uma visão construtivista. In. E. Boruchovich, J. A. Buzneck (orgs). Aprendizagem: processos psicológicos e contextos social na escola (pp. 17-54). Petrópolis: Vozes.

Boruchovitch, E. (1999). Estratégias de aprendizagem e desempenho escolar: considerações para a prática educacional. Psicologia: Reflexão e Crítica. Porto Alegre, v.12, n. 2, p. 361-376.

Boruchovitch, E. (2007). Aprender a aprender: propostas de intervenção em estratégias de aprendizagem. ETD Educação Temática Digital, Campinas, v.8, n.2, p. 156167, jun.

Corno, L. (2001). Volitional aspects of self-regulated learning. In. B. J. Zimmerman \& D. H.

Carta, L.F.L.(2012). Avaliação do uso de estratégias volitivas: estudo exploratório utilizando o método QSORT em jovens do $7^{\circ}, 8^{\circ}$ e $9^{\circ}$ ano de escolaridade. Mestrado integrado em Psicologia. Universidade de Lisboa, Lisboa, Portugal.

Schunk (Eds). Self-Regulated Learning and Academic Achievement. Mahawh,.( pp. 191-225).New Jersey: Laurence Erlbaum Associates, Inc., Publishers

Deps, Vera L.; Veiga Simão, A. M.; Lopes Da Silva, A. (2011). A construção de um instrumento de estratégias volitivas pelo método Q-sort. Rio de Janeiro: EDUENF.

Frison, L. M. B.; Veiga Simão ; Nonticuri, A. (2015) . Dos significados à autorregulação: perspectivas de estudantes com trajetórias acadêmicas de insucesso. Revista de Estudios e Investigación en Psicología y Educació, v. Extr 1, p. 048-053.

Medeiros, S. (2014). Avaliação do uso de estratégias do controlo do desempenho em estudantes do $3^{\circ}$ ciclo do Ensino Básico. Mestrado integrado em Psicologia. Universidade de Lisboa, Lisboa, Portugal.

Miranda, C. A. G. R.; Nonticuri, A. R.; Louzada, R. S; Frison, L. M. B. (2016). Quais estratégias os estudantes universitários consideram importantes para a aprendizagem? In: XXV Congresso de Iniciação Científica, 2016, Pelotas. Anais do XXV Congresso de Iniciação Científica, v. 15. p. 1-4.

Perassinato, M. G. M.; Boruchivich, E.; Oliveira, K.L. (2012). Estratégias De Aprendizagem no Ensino Fundamental: Revistando Instrumentos de Medida. In: Boruchovitch, E.; Santos. A. A. A.; Nascimento, E. Avaliação psicológica nos contextos Educativos e Psicossocial. (pp. 53- 78). São Paulo: Casa do Psicólogo.

Pozo, J. I. (1998). Teorias Cognitivas da Aprendizagem. $3^{\mathrm{a}}$ Ed. Porto Alegre: Artmed. 
Rosa, G.P. Deps, V. L. (2015) Desatenção do aluno e estratégias de aprendizagem no contexto escolar. Revista Científica Interdisciplinar. p. 247 de 260.

Serafim, T. M.; Boruchovitch, E. (2010). O Pedir Ajuda: Concepções dos Estudantes do Ensino Fundamental. Est. Inter. Psicol., Londrina , v. 1, n. 2, p. 159-171, jun.

Silva, A. L., Veiga Simão, A. M. Sá, I. A (2004). Autoregulação da Aprendizagem: Estudos Teóricos e Empíricos. Intermeio: revista do Mestrado em Educação, Campo Grande, MS, v. 10, n. 19, p. 58-74.

Schunk, D. H. (1989). Self efficacity and academic motivation. Educational Psychologist, $26(3,4)$, p. $207-$ 231.

Veiga Simão, A. M. (2004) O conhecimento estratégico e a auto-regulação da aprendizagem: implicações em contexto escolar. In: LOPES DA SILVA, A; DUARTE, A. M; SÁ, I; VEIGA SIMÃO, A. M. (Orgs.). Aprendizagem auto-regulada pelo estudante: perspectivas psicológicas e educacionais. (p.77-94). Porto: Porto Editora.

Veiga Simão, A. M. (2013). Ensinar para a aprendizagem escolar. In: VEIGA. F. (Org.) Psicologia da Educação: teoria, investigação e aplicação. (p. 495-541). Lisboa: Climepsi Editores.

Veiga Simão, A. M.; Frison, L. M. (2013) Autorregulação da aprendizagem: abordagens teóricas e desafios para as práticas em contextos educativos. Cadernos de Educação, Pelotas, v.45, p. 02-20.

Veiga Simão, A. M.; Lopes da Silva, A. A (2012). Construção de um Instrumento de Estratégias Volitivas pelo Método Q-SORT. In. Lyra. P. (Org) Conhecimento em Processo. Ensaios Interdisciplinares sobre Linguagem e Cognição. Rio de Janeiro: Ed. Tempo Brasileiro EDUENF, p. 221-242.

Veiga Simão, A. M.; Lopes Da Silva, A. (2012). A Construção de um Instrumento de Estratégias Volitivas pelo Método Q-SORT. In P. Lyra (Org) Conhecimento em Processo. Ensaios Interdisciplinares sobre Linguagem e Cognição (pp. 221-242). , Rio de Janeiro: Ed. Tempo Brasileiro EDUENF

Zimmerman, B. J. (1989). Models of self-regulated learning and academic achievement. In, B.J Zimmerman \&, D.H. Schunk (Eds), Self-Regulated Learning and Academic Achievment. Theory, research and Practice. Progress in Cognitive Development Research. (pp.1-26). New York: Springer-Verlag.

Zimmerman, B. (1998). Developing self-fulfilling cycles of academic regulation: na analysis of exemplary instructional models. In. SCHUNK, D.; ZIMMERMAN, B. Self-regulated learning: from teaching to self-reflective practice. (p.1-19). New York: The Guilford Press

Zimmerman, B. J., \& Martinez-Pons, M. (1986). Development of a structured interview for assessing student use of self-regulated learning strategies. American Educational Research Journal, 23(4), 641628.

Zimmerman, B. J. (2000). Attaining self-regulation: A social cognitive perspective. In $\mathrm{M}$. Boekaerts, $\mathrm{P}$. Pintrich e M. Zeidner (Eds.), Handbook of SelfRegulation. New York: Academic Press, p. 13-39.
Zimmerman, B. (2013). From cognitive modeling to selfregulation: a social cognitive career path. Journal of Educational psychologist, Washington, v.48, n.3, p.135-147.

\section{Agradecimentos}

Esta pesquisa foi financiada pelo Conselho Nacional de Desenvolvimento Científico e Tecnológico - CNPQ. 